\title{
Effects of constraint and validity of sentence contexts on lexical decisions
}

\author{
IRA S. FISCHLER and PAUL A. BLOOM \\ University of Florida, Gainesville, Florida
}

\begin{abstract}
Recent models of sentence context effects predict that the pattern of facilitation and inhibition of response to sentence completions should be influenced by the experiment-wide contextual "environment." In the present experiments, this environment was manipulated in several ways, including the degree to which contexts constrained possible completions, the probability of predictable completions' being presented, and the probability of congruous completions' being presented. In Experiment 1, decreasing the proportion of congruent test words had no effect on either the facilitation for highly likely words or the inhibition for incongruent words; increasing the proportion of predictable words produced no increase in facilitation for these words, but did increase the inhibition for incongruous words. In Experiment 2, contexts with very high or very low degrees of constraint produced equivalent results when predictability was uniformly low: no facilitation for unlikely but congruent words, and inhibition for incongruent words. In general, the patterns of change in facilitation and inhibition caused by changes in the contextual environment were more consistent with the modified two-process model (Stanovich \& West, 1983) than with the verification model (Becker, 1982). But the limited range of influence suggests that, under conditions approximating normal reading, little use is made of such "metacontextual" information.
\end{abstract}

It is not difficult to demonstrate that sentence contexts can influence performance on a variety of word processing tasks. But the mechanism of this influence, as well as its corresponding implications for theories of word retrieval and of reading, remains controversial. The present experiments concerned the extent to which a reader's expectancies can determine these context effects. Our approach was to vary systematically the information provided by sentence contexts at an "experiment-wide" level in several distinct ways. Recent models of the effects of verbal contexts make contrasting predictions about how changes in the contextual "environment" should influence the pattern of contextual effects.

The basic paradigm involves presentation of an incomplete sentence context followed by a word that completes or continues the sentence. In most cases, some kind of neutral condition is used to separate context effects into facilitation-the advantage gained by presenting a test word that is congruous with the context-and inhibitionthe costs of incongruous test words.

Schuberth and Eimas (1977) found both facilitation for congruous words and inhibition for incongruous words on lexical decision latency. Similar results were obtained by Fischler and Bloom (1979), who also showed that the amount of facilitation was positively related to the predictability of congruous words when predictability varied

This research was reported in part at the meeting of the Midwestern Psychological Association, Detroit, May 1981. We are grateful to Keith Stanovich and an anonymous reviewer for their helpful comments on the manuscript. Paul Bloom is now with Tropicana Co., Bradenton, FL. Requests for reprints should be sent to Ira Fischler, Psychology Department, University of Florida, Gainesville, FL 32611. within subjects over a wide range. The inhibition has been shown to be a robust finding for lexical decision latency (see Fischler, 1985, Fischler \& Bloom, 1980, Sanocki \& Oden, 1984, Schwanenflugel \& Shoben, 1985, Experiment 4, and Stanovich \& West, 1983, Experiment 11).

According to the two-process model of Posner and Snyder (1975), cue stimuli can influence subsequent performance by the separate or additive processes of automatic pathway activation and attentional expectancies. According to the model, facilitation can be produced by either process, but attentional facilitation is accompanied by inhibition for unexpected tests. The inhibition of lexical decision latency, by this logic, suggests that the context is used to form expectancies about possible test words, and the occurrence of incongruous test words involves redirection of attention, slowing the response.

Early support for the two-process model came from a series of studies by Stanovich and West $(1979,1981)$ showing that, when a naming task was used, facilitation was obtained in the absence of inhibition. It was argued that, for adult readers, naming a word was a highly overlearned and rapid response, one that occurred too fast for conscious expectancies to influence naming speed. Conversely, the facilitation was caused by an automatic spread of activity from words or concepts in the context related to the test word.

Other studies by Stanovich and West provided further support for this interpretation. First, facilitation of naming is greater, and inhibition often becomes significant, for less skilled readers, for whom word recognition is less automatic (Stanovich, West, \& Freeman, 1981; West \& Stanovich, 1978; cf. Fischler, 1985). Facilitation and inhibition are also increased by using words that are 
difficult to recognize in isolation (Stanovich \& West, $1981,1983)$, by making the test word more difficult to see (Stanovich \& West, 1979, 1983, Experiment 6; but cf. Experiments 7 and 8 ), and by increasing the delay between the last word of the context to be read and the presentation of the word to be named (Stanovich \& West, 1981, Experiment 2).

Becker (1980; Eisenberg \& Becker, 1982) has presented an alternative explanation for the effects of sentence contexts. In his verification model, a semantic context elicits a set of candidate words against which the test stimulus can be matched following preliminary sensory analysis. If the test matches a word in the set, recognition can occur without further visual analysis. Becker states that if the semantic set contains few items, the matching process will take little time. A match will therefore be fast, whereas failure to match will have little effect on response time. The overall pattern, then, should be one of large facilitation for predicted words and little or no inhibition for other items. If the set is large, however, the advantage for matches will be diminished, and there will be little facilitation. Test words not in the large set will always require an exhaustive search of the semantic set, resulting in slower decision times than will trials with no context. The overall pattern in this case will be of inhibition dominance.

The respective successes and failures of two-process theory and the verification theory to explain the basic facts of contextual effects have been discussed elsewhere (Eisenberg \& Becker, 1982; Schuberth, Spoehr, \& Lane, 1981; Stanovich \& West, 1983). The focus of the present experiments is on how well these two models predict the effects of changes in the contextual environment-which presumably influence the expectations a subject may entertain as the experiment progresses-on the pattern of lexical decision latencies for various test items.

As Schwanenflugel and Shoben (1985) recently pointed out, these expectancies could concern any or all of three different kinds of information about the contexts and test items: (1) The constraint of the context-To what extent does the sentence context suggest one likely completion? This is usually quantified as the probability of the dominant, or most frequent, response to the context in a completion task. (2) The predictability of the test word-How likely is it that a particular test word will be presented? Note that with high-constraint contexts, overall predictability can be high or low, depending on the proportion of primary responses actually presented across trials. With low-constraint contexts, in contrast, predictability of particular completions is necessarily low. (3) The congruity of context and test item-What proportion of sentence contexts are followed by words that are plausible, or congruous, in that context?

Each of these forms of contextual information has varied widely across the studies of sentence contexts effects reviewed above, but there have been few direct manipulations of the experiment-wide environment. It is possible that at least some of the inconsistencies across experi- ments are due to these variations in experiment-wide validity. For example, the use of a small number of highconstraint contexts and predictable completions in Fischler and Bloom (1979) may have caused a narrowing of expectations, resulting in the absence of any facilitation for low-constraint, low-predictability congruous contexts that are most typical of normal reading materials.

Fischler and Bloom (1979) did include one extreme manipulation of the contextual environment. In their Experiment 2 , the inhibition for incongruous word completions observed in their first experiment was eliminated by presenting no congruous completions at all. Although this shows that the absence of context validity can eliminate a context effect, the manipulation is not representative of the mixtures of validity used in other studies. Also the critical comparison was made across experiments.

Stanovich and West $(1981,1983)$ have shown that the proportion of congruous trials (.33 vs. .66) did not alter the pattern of context effects on naming latency, which showed significant facilitation for congruous trials and litthe inhibition for incongruous trials. This was true for contexts providing high constraint (1981, Experiment 1) and low constraint (1983, Experiment 4; average predictability for congruous completions $=.11$ ). With the proportion of congruous tests controlled, Stanovich and West (1983, Experiment 3) also found little effect of whether relatively more predictable (mean $\mathrm{p}=.43$ ) or less predictable (mean $p=.11$ ) words were presented on congruous trials in the first half of the experiment.

The absence of systematic effects of context validity on naming latency is consistent with Stanovich and West's (e.g., 1983) contention that naming a word is a relatively automatic and autonomous process that will not normally be influenced by expectations. In single-word priming experiments, it appears that both the proportion of relatedprime trials (den Heyer, Briand, \& Dannenbring, 1983; Tweedy, Lapinski, \& Schvaneveldt, 1977) and the predictability of targets given related primes (antonyms vs. category exemplars; Becker, 1980) influence lexical decision latency, whereas the proportion of related trials has no influence on naming latency (Seidenberg, Waters, Sanders, \& Langer, 1984). Seidenberg et al. (1984), West and Stanovich (1982), and others have stressed the importance of task type in explaining the variety of results in studies of how sentence contexts affect word recognition. The lexical decision, it is argued, can be biased both by subject expectancies and by the postaccess congruity of context and test item (see Forster, 1979). The latter process allows for a Stroop-like incongruity effect on lexical decision that could be obtained in the absence of either sufficient time or motivation to develop the sort of expectancies needed to produce attentional inhibition.

The purpose of the present experiments was to see what, if any, effect variations in experiment-wide contextual environment similar to those used in the naming experiments of Stanovich and West $(1981,1983)$ would have on lexical decision latency. Both the verification model and the 
two-process model suggest that validity will influence lexical decision latency, although in very different ways (see below). In contrast, in their recent discussion of such expectancy effects, Stanovich and West (1983) suggested that comprehension of sentences utilizes the same limited resources that subjects use to generate expectancies in the single-word priming studies, and therefore, although lexical decision may be more sensitive to such expectancies, cue validity of sentence contexts will have little or no effect because subjects will not be able to generate or use these expectancies. Their modified two-process model suggests that inhibition will be limited to the relatively automatic, postaccess incongruity effect that will not be eliminated by low contextual validity.

It should be noted that the term "expectancy" here concerns the ability of subjects to monitor the experimentwide relations between context and test items, and to use this information in making lexical decisions. It is a more general sense of expectations than is sensitivity to the predictability of individual context-test pairings (see Becker, 1980, pp. 494-495).

In contrast to our earlier studies of sentence context effects, several changes were made in the general procedure that were intended to enhance any use of expectancies. First, the proportion of word trials was increased from .50 to .60 . Second, nonwords that resembled highly likely, congruous words (e.g., The children went outside to BLAY) were avoided. Third, since the facilitation in Fischler and bloom (1979) was confined to contexts that provided a great deal of constraint (above .80 ), a larger set of such highly constraining contexts was developed (Bloom \& Fischler, 1980, Appendix B) to provide a stronger manipulation of constraint and predictability.

In Experiment 1, congruity and predictability were manipulated while contextual constraint was held constant at a high level. In Experiment 2, constraint was varied while congruity and predictability were held constant. In each study, all subjects were asked a number of questions about the validity of the contexts at the end of the session. We were interested in how accurately subjects could assess several aspects of sentence validity in their condition, and the extent to which this knowledge correlated with the pattern of facilitation and inhibition observed.

\section{EXPERIMENT 1}

In the first experiment, the sentence contexts were all highly constraining, with a mean probability of the most likely completion of .85 . When a sentence context was presented, probability of a word test was .6. This word could be the most likely completion, an unlikely but congruous completion, or an incongruous completion. The proportion of these three types of completions was varied across three groups, as shown in Table 1 . In the balanced group, each type of word test was equally likely to be presented. In the high-predictability environmnent, all the congruous word tests were the likely completions. Here, congruity was the same as for the balanced environment, but the average predictability was increased substantially. Finally, for the low-congruity group, the unlikely completions were replaced by incongruous completions, making incongruous completions twice as likely as congruous ones. Note that the predictability of specific words for the low-congruity group was essentially equal to that of the balanced group.

Predictions based on the two-process model will be considered first. Since a lexical decision task is used, and the range of various word completions is similar to that in Fischler and Bloom (1979), both facilitation for likely words and inhibition for incongruous words was expected. Since the constraint of the present sentence contexts is generally much higher, this should lead to greater use of attentional strategies than was observed in our previous studies, and this should enhance both the facilitation and inhibition effects. It also may lead to significant inhibition of response to the unlikely test words, a tendency that did not reach significance in Fischler and Bloom (1979).

Similarly, both the facilitation of likely test words and the inhibition of incongruous test words should be greatest for the high-predictability condition, since the greater proportion of sentences completed by a very predictable word should lead to greater attentional effects. In contrast, decreasing the proportion of congruous tests in the session may reduce the use of contextual information, decreasing both the facilitation and the inhibition.

An important characteristic of the predictions of the two-process model, then, is that any correlation of facilitation and inhibition across conditions should be positive; no manipulation of expectancy should increase one while decreasing the other. A similar prediction can be made across subjects within a given condition, and has been the basis for some of the tests of two-process versus verification models. The correlation of facilitation and inhibition across subjects within a condition could be assessed, since a separate set of neutral trials corresponding to each context condition was run.

The modified version of the two-process model (West \& Stanovich, 1982) predicts, first, that any withinenvironment correlation of facilitation and inhibition should be greater for the more valid environments, in which expectancies are more likely, and, second, that even in low-validity conditions, both facilitation (due to associative priming effects) and inhibition (due to the Strooplike incongruity effect) should still be present, but may be uncorrelated.

The verification model makes somewhat different predictions about context effects in these conditions. If the critical factor is constraint-the degree to which the context allows particular words to be predicted and thus included in the semantic verification set-then other aspects of the contextual environment should have little impact on use of context. In no case should inhibition be observed, since the semantic set can be small with the highly constraining contexts. Facilitation should be observed for likely words in each of the three environments. 
If, however, decreasing the predictability or congruity of completions has the effect of shifting subjects to an "expectancy" strategy, in which the semantic set contains many words (Becker, 1980), then the amount of facilitation should be greatest and inhibition smallest for the highpredictability environment, and, conversely, facilitation smallest and inhibition greatest for the low-congruity environment. Within an environment, there is also variation in the strategy adopted by subjects, so that any correlation of facilitation and inhibition scores across subjects within a group should be negative.

In addition to these specific predictions, both models suggest that expectancy effects should increase as subjects become familiar with the overall contextual environment. A modest test of this was made possible by including two successive pairs of context and neutral trial blocks.

\section{Method}

\section{Stimulus Materials}

A set of 100 sentence contexts, drawn from the norms of Bloom and Fischler (1980), had a probability of the primary completion of at least .79 (mean $p=.85$ ). Each sentence was associated with three kinds of word completion. The most frequent completion listed in Bloom and Fischler served as the likely word. A word whose probability of completion was less than .20 (mean $p=.01$ ) was selected as the unlikely completion for that sentence. Forty percent of these words were actual responses from the norms; the remainder were judged by two independent raters as being congruous or plausible completions of the sentences. A word that was judged implausible in that context served as the incongruous word. Mean word length and number of syllables were roughly balanced across the three kinds of word tests. Mean frequency of occurrence (Kučera \& Francis, 1967) was as follows: likely words, 211 per million; unlikely words, 166 per million; and incongruous words, 178 per million.

Each sentence was also associated with two nonword stimuli. These were generated by changing a single letter of the unlikely and incongruous words for that sentence so that the resulting string was a pronounceable pseudoword.

\section{Design}

Context type (incomplete sentence or no context) and test-item type (likely, unlikely, incongruous, or nonword) were varied within subjects. Three groups were defined by the proportion of the three types of word trials given during the session. For all subjects, a 6:4 ratio of words to nonwords was maintained in each block of trials. On word trials, the subjects in the balanced environment received an equal proportion of likely, unlikely, and incongruous tests. For the high-predictability group, the unlikely words were replaced by the corresponding likely words, making all the con- gruous completions very predictable. For the low-congruity group, the unlikely words were replaced by the corresponding incongruous words, making two-thirds of the word tests incongruous. The proportion of test types per condition is outlined in Table 1.

Over the single session of 200 trials, each of the subjects saw two of the test items associated with a given context; one was preceded by that context, and the other was preceded by a string of Xs. ${ }^{1}$ These sentence-context and no-context trials were presented in alternating blocks of 50 trials each. Half the subjects received a sentence-context block first. The 100 sets of contexts and test items were divided into five subsets and assigned to the five test-item conditions (three word and two nonword tests) across subjects according to a Latin square. In the balanced environment, this meant that, over every five subjects, each test item was seen once in the sentencecontext condition and once in the no-context condition. For the highpredictability environment, each likely word was seen twice every five subjects, and for the low-congruity environment, each incongruous word was seen twice every five subjects.

\section{Apparatus}

Stimuli were presented on a VT100 display terminal with a 16.7msec refresh cycle, driven by a PDP-11/34 computer. Characters were written white on black in a $7 \times 9$ dot matrix, with a writing time of approximately 1 character/msec. Effective visual height of the characters was about $1^{\circ}$.

\section{Procedure}

At the start of the session, the nature of the lexical decision task was explained, and the following points were made about the relation of sentence contexts and test items: (1) that all words would be familiar, so that the emphasis was on "how quickly words could be recognized as words, and how different kinds of context affect decision time;" (2) that on sentence-context trials, there would sometimes be test words that made "no sense of the context," but still required a positive response; and (3) that on sentence-context trials, the contexts should be "read and understood," and that on no-context trials, an effort should be made to visually scan the row of Xs when they appeared. Nothing was said about the predictability of words or the constraint of the sentences on sentence-context trials

Each trial consisted of the following display sequence: an asterisk shown at the center of the display for $0.5 \mathrm{sec}$; a blank interval of $0.5 \mathrm{sec}$; a sentence context or string of Xs for $2 \mathrm{sec}$; another 0.5 -sec blank interval; and a word or nonword that remained visible until a subject had made a response by pressing one of two letters on the terminal keyboard with the index finger of one hand. Assignment of hand to response was counterbalanced across subjects. The string of Xs had the same number of characters and arrangement of blank spaces as the corresponding sentence context for the test item that followed. Sentence contexts were shown in lowercase, and test items in uppercase. Response accuracy and latency were recorded on-line, and 3 sec later the next trial began.

Following completion of the fourth block of trials, the subjects were asked to estimate two aspects of the validity of the materials

Table 1

Composition of Sentence Context Trials for the Different Validity Conditions of Experiments 1 and 2

\begin{tabular}{clccccc} 
& & \multicolumn{4}{c}{ Proportion of Lexical Decision Trials } \\
\cline { 3 - 6 } Experiment & Validity Group & Constraint & Likely & Unlikely & Incongruous & Nonwords \\
\hline 1 & Balanced & High & .2 & .2 & .2 & .4 \\
1 & High-predictability & High & .4 & .0 & .2 & .4 \\
1 & Low-congruity & High & .2 & .0 & .4 & .4 \\
2 & High constraint & High & .0 & .4 & .2 & .4 \\
2 & Low constraint & Low & .0 & .4 & .2 & .4 \\
\hline
\end{tabular}


they saw during the lexical decision task. The first question concerned the congruity of sentences and tests, and was phrased as follows: "On trials where words were shown as test items following sentence contexts, what proportion of these words seemed to make sense of the sentence context?" The second question concerned the predictability of test words, and was phrased thus: "On those trials where words were shown as test items following sentence contexts, what proportion of tests were words that you think you particularly expected as a completion?"

\section{Subjects}

A total of 60 students enrolled in general psychology at the University of Florida served in the experiment, 20 in each environment condition, in partial fulfillment of the course research requirement. Students were assigned to groups according to the order of appearance in the laboratory.

\section{Results}

Mean latency of correct responses was obtained for each subject in each context/test-item condition, and was used as a single score in the analyses that follow. Separate analyses of variance were performed on data from word test and nonword test trials. Effects described as significant are so at a .05 probability level, with a .01 probability level adopted for subsequent tests of simple effects.

\section{Balanced Condition}

Because the balanced group was the only one to include unlikely words as test items, separate analyses were first performed on the data for this group. The ANOVA included context type (sentence or neutral), test-item type (likely, unlikely, or incongruous), and trial block as within-subject factors. Mean latencies and error rates for each condition are shown in Table 2 .

Responses to words. The differences among means was reflected in a significant three-way interaction of context type, test type, and block $[F(2,38)=4.82]$. Tests of the simple effects of context type showed a significant facilitation for likely words in both blocks, a significant inhibition for incongruous words in both blocks, and no effect of context in either block for the unlikely words. The interaction of the context effects with block is seen in the somewhat larger facilitation and substantially smaller inhibition for the second than for the first block. Latencies overall were somewhat faster in the second block $[F(1,19)$ $=17.49]$.

Differences among the error rates mirrored the pattern for latencies, with sentence contexts producing fewer errors for likely words, greater errors for incongruous words, and little effect on errors to unlikely words. An ANOVA for the arcsin-transformed error rates was performed. The interaction of context and test type was significant $[F(2,38)=10.43]$. With error rate for neutral contexts very low, the small facilitation for likely words in context was not significant, in contrast to the substantial contextual inhibition for incongruous words $(9.3 \%$ vs. $1.3 \%)$.

Responses to nonwords. Latency for correct responses to nonwords was also analyzed for context effects. Although latencies were somewhat faster for sentencecontext trials ( 648 vs. $668 \mathrm{msec}$ ), the difference was not significant $[F(1,19)=4.32, p<.10]$. In contrast to the data for word trials, responses to nonwords were not significantly faster in the second than in the first block . $[\mathrm{F}(1,19) \mathrm{p}<1.00]$. Error rates for nonwords were marginally lower for sentence context trials $(5.4 \%$ vs. $8.5 \%)$ $[\mathrm{F}(1,19)=1.78, \mathrm{p}>.10]$.

\section{Comparisons Across Validity Groups}

Responses to words. A mixed-model ANOVA was performed on the latencies of correct responses to word tests for likely and incongruous word types across the three environments. Main effects for environment, context type, test type, and blocks were obtained, but each factor was involved in higher order interactions. The first of interest was a significant interaction of environment, context type, and test type $[F(2,57)=6.58]$. The nature of this interaction is seen in the bottom row of Table 2, comparing the absolute magnitude of facilitation (likely word tests) and inhibition (incongruous word tests) across condition. Simple effects showed contextual facilitation to be sig-

Table 2

Mean Lexical Decision Latency (in Milliseconds) and Error Rates (ERs) for Three Validity Environments of Experiment 1

\begin{tabular}{|c|c|c|c|c|c|c|c|c|c|c|c|c|c|c|}
\hline & \multicolumn{14}{|c|}{ Validity Environment } \\
\hline & \multicolumn{6}{|c|}{ Balanced } & \multicolumn{4}{|c|}{ High Predictability } & \multicolumn{4}{|c|}{ Low Congruity } \\
\hline & \multicolumn{2}{|c|}{ Likely } & \multicolumn{2}{|c|}{ Unlikely } & \multicolumn{2}{|c|}{ Incongruous } & \multicolumn{2}{|c|}{ Likely } & \multicolumn{2}{|c|}{ Incongruous } & \multicolumn{2}{|c|}{ Likely } & \multicolumn{2}{|c|}{ Incongruou } \\
\hline & Mean & ER & $\overline{\text { Mean }}$ & ER & Mean & $\overline{E R}$ & Mean & $\overline{E R}$ & Mean & $\overline{E R}$ & Mean & ER & $\overline{\text { Mean }}$ & ER \\
\hline $\begin{array}{l}\text { Block I } \\
\text { Context } \\
\text { Neutral }\end{array}$ & $\begin{array}{l}4790 \\
521\end{array}$ & $\begin{array}{l}0.5 \\
2.0\end{array}$ & $\begin{array}{l}546 \\
540\end{array}$ & $\begin{array}{l}4.0 \\
2.5\end{array}$ & $\begin{array}{l}636 \\
538\end{array}$ & $\begin{array}{r}10.5 \\
1.5\end{array}$ & $\begin{array}{l}555 \\
589\end{array}$ & $\begin{array}{l}2.0 \\
3.0\end{array}$ & $\begin{array}{l}748 \\
597\end{array}$ & $\begin{array}{r}11.0 \\
2.5\end{array}$ & $\begin{array}{l}501 \\
529\end{array}$ & $\begin{array}{l}2.5 \\
2.5\end{array}$ & $\begin{array}{l}630 \\
543\end{array}$ & $\begin{array}{l}6.5 \\
2.8\end{array}$ \\
\hline Context Effect & \multicolumn{2}{|c|}{42} & \multicolumn{2}{|c|}{-6} & \multicolumn{2}{|c|}{-98} & \multicolumn{2}{|c|}{34} & \multicolumn{2}{|c|}{-151} & \multicolumn{2}{|c|}{28} & \multicolumn{2}{|c|}{-87} \\
\hline $\begin{array}{c}\text { Block } 2 \\
\text { Context } \\
\text { Neutral }\end{array}$ & $\begin{array}{l}461 \\
511\end{array}$ & $\begin{array}{l}0.5 \\
1.0\end{array}$ & $\begin{array}{l}534 \\
526\end{array}$ & $\begin{array}{l}3.0 \\
2.5\end{array}$ & $\begin{array}{l}568 \\
522\end{array}$ & $\begin{array}{l}8.0 \\
1.0\end{array}$ & $\begin{array}{l}500 \\
575\end{array}$ & $\begin{array}{l}2.0 \\
2.5\end{array}$ & $\begin{array}{l}677 \\
584\end{array}$ & $\begin{array}{r}11.1 \\
2.5\end{array}$ & $\begin{array}{l}463 \\
519\end{array}$ & $\begin{array}{l}1.0 \\
4.0\end{array}$ & $\begin{array}{l}565 \\
530\end{array}$ & $\begin{array}{l}6.0 \\
2.8\end{array}$ \\
\hline Context Effect & \multicolumn{2}{|c|}{50} & \multicolumn{2}{|c|}{-8} & \multicolumn{2}{|c|}{-46} & \multicolumn{2}{|c|}{75} & \multicolumn{2}{|c|}{-93} & \multicolumn{2}{|c|}{56} & \multicolumn{2}{|c|}{-35} \\
\hline Mean Effect & \multicolumn{2}{|l|}{46} & \multicolumn{2}{|c|}{-7} & \multicolumn{2}{|c|}{-72} & \multicolumn{2}{|l|}{54} & \multicolumn{2}{|c|}{-122} & \multicolumn{2}{|c|}{42} & \multicolumn{2}{|c|}{-61} \\
\hline
\end{tabular}

Note-Context effect is neutral minus context latency, so a negative effect indicates contextual inhibition. 
nificant in all three groups, with the absolute magnitude of facilitation somewhat greater for the high-predictability condition ( $54 \mathrm{msec}$ ) and lower for the low-congruity condition ( $42 \mathrm{msec}$ ) than for the balanced group $(46 \mathrm{msec})$. An analysis of the facilitation scores by subject showed that the magnitude of facilitation did not differ across the three groups. Significant contextual inhibition was also found for incongruous words in each group, and was significantly larger for the high-predictability group (122 $\mathrm{msec})$ than for either the balanced $(72 \mathrm{msec})$ or lowcongruity $(61 \mathrm{msec})$ groups, which did not differ from each other (Newman-Keuls test of the inhibition scores by subject).

A second three-way interaction involved context, test type, and block $[F(1,57)=4.18]$. Across conditions, this can be seen as the tendency for facilitation to increase (32 vs. $60 \mathrm{msec}$ across groups) and inhibition to decrease (112 vs. $58 \mathrm{msec}$ ) from the first to the second block. Although the absolute size of the shift varied across groups, the fourway interaction with environment was not significant $[\mathrm{F}(2,57)<1.00]$.

The pattern of error rates for the high-predictability and low-congruity conditions was very similar to that for the balanced group, and in the ANOVA performed on arcsintransformed error rates, no effect involving environment approached significance. As before, the context type $\times$ test type interaction was significant $[F(1,57)=51.84]$. Simple effect tests showed that the inhibition effect on error rates $(9.0 \%$ vs. $2.2 \%)$ was significant, but that the facilitation effect $(1.4 \%$ vs. $2.5 \%)$ was not.

Responses to nonwords. For latencies to nonwords, the ANOVA showed a significant context type $\times$ environment interaction $[F(2,57)=3.91]$. Similar to the balanced group, the high-predictability group showed somewhat faster responses to nonwords following sentence contexts ( 685 vs. $716 \mathrm{msec}, \mathrm{p}<.02$ ), but for the low-congruity group, latencies following sentence contexts were nonsignificantly lower (676 vs. $662 \mathrm{msec}$ ). For error rates, the context type $\times$ validity interaction was not significant; overall, however, more errors were made following sentence contexts $(7.8 \%)$ than following neutral contexts $(5.5 \%)[\mathrm{F}(1,57)=7.82]$, indicating at least a modest speed-accuracy trade-off for nonword performance across groups.

\section{Individual Differences in Facilitation and Inhibition}

The magnitude of facilitation for each subject was the difference in response latency for sentence-context and neutral-context trials for likely words, as above. Individual inhibition scores were similarly obtained for the incongruous words. The measure of interest was the correlation of these two measures across subjects for each group. In each case, the direction of the correlation was negative, with less facilitation associated with greater inhibition (balanced $r=-.31$; high predictability, -.08 , low congruity, -.26 ), but none of the correlations was significant.

For the balanced group only, a third contextual-effect score was computed for each subject for the unlikely words in sentence versus neutral contexts. Although there was no overall effect for these test words (6-msec inhibition), the range across subjects was sizable, from a 68$\mathrm{msec}$ facilitation to a 76-msec inhibition. Nonetheless, these unlikely word-context scores were uncorrelated with either the likely-word facilitation $(\mathrm{r}=+.12)$ or the incongruous-word inhibition $(r=-.07)$.

\section{Subject Reports of Validity}

Subjects' post hoc estimates of the proportion of congruent- and predictable-word tests are given in Table 3 , along with the actual proportions, for each environment condition. Given that there may have been some variability in how subjects interpreted these questions, it is clear that, at least on an ordinal basis, subjects estimate the actual probabilities of congruous and predictable completions fairly well. Congruence judgments were higher for the balanced and high-predictability groups than for the low-congruity groups $[F(2,57)=13.11]$. A Newman-Keuls test showed that the estimates of the highpredictability group were not different from those of the balanced group $(\mathrm{q}=2.53, \mathrm{p}>.05)$.

Estimated proportion of predictable tests was also in the correct order across groups, but the differences did not reach significance $[F(2,55)=2.73, p<.10]$.

\section{Discussion}

In all three environments, the presence of sentence contexts preceding a lexical decision speeded responses to highly predictable words and slowed responses to incongruous words. Also consistent with our previous studies, sentence contexts had little effect on latency to congruous but unlikely words, suggesting that the "congruity effect" is more an "incongruity effect," and that, at least when the series of trials contains some highly predictable test words, congruity per se provides no facilitation of decision latency.

Table 3

Subjects' Estimates of the Proportion of Congruous and of Predictable Word Tests in Experiment 1

\begin{tabular}{|c|c|c|c|c|}
\hline & & \multicolumn{3}{|c|}{ Validity Environment } \\
\hline & & Balanced & High Predictability & Low Congruity \\
\hline Probability of words that were congruous in context & $\begin{array}{l}\text { Reported } \\
\text { Actual }\end{array}$ & $\begin{array}{l}.48 \\
.67\end{array}$ & $\begin{array}{l}.58 \\
.67\end{array}$ & $\begin{array}{l}.30 \\
.33\end{array}$ \\
\hline Predictability of words in context* & $\begin{array}{l}\text { Reported } \\
\text { Actual }\end{array}$ & $\begin{array}{l}.34 \\
.28\end{array}$ & $\begin{array}{l}.48 \\
.57\end{array}$ & $\begin{array}{l}.32 \\
.28\end{array}$ \\
\hline
\end{tabular}

*Probability of likely word given word $\times$ cloze probability of the likely word. 
The main questions were whether substantial changes in the proportion of predictable and congruous test words could be detected by subjects, and, if so, how these changes might affect the pattern of facilitation and inhibition. The subjects' responses to the postsession questions clearly showed that they could fairly accurately estimate the proportion of congruence and, to a lesser degree, predictability of the test words in context, so that information about these aspects of contextual validity was derived during the session. The effect of context validity on decision latency, however, was limited. Compared with the balanced condition, increasing the probability of a predictable completion in the high-predictability group resulted in a sizable increase in inhibition for incongruous test words. Facilitation was also increased, but only by a nonsignificant $8 \mathrm{msec}$. Conversely, decreasing the probability of a congruous test in the low-congruity condition produced an insignificant reduction in the facilitation and inhibition effects.

These results are more consistent with predictions of the two-process model than with those of the verification model. First, the magnitude of facilitation and inhibition tended to vary directly across environment, rather than inversely. Second, the condition that should lead to greater use of expectancies about upcoming test items (the high predictability condition) shows the greatest amount of these contextual effects, and the low-congruity condition, in which subjects should have least motivation for making use of contextual information, shows the smallest amount of contextual effects.

Several other aspects of the data are consistent with the basic idea of two-process theory, that is, that there are two distinct processes that mediate contextual effects. In general, facilitation for predictable words was smaller than inhibition for incongruous words, and was much less influenced by changes in experiment-wide validity. This would be the case if the facilitation were due in large part to an automatic priming of highly likely target words. Since the occurrence of word-level associations between context and test was not completely avoided (See Bloom \& Fischler, 1980), at least part of this facilitation may come from such lexical associative activation (see Stanovich \& West, 1983, for an estimate of the contribution of sentence-level priming to the facilitation; see also Fischler \& Bloom, 1980, pp. 221-222, and Foss, 1982). It is also interesting that, in general, the subjects seemed to be less sensitive to the degree of predictability than to the degree of congruence in their verbal reports.

The independence of the magnitude of facilitation and inhibition across subjects within each condition also suggests two distinct processes. The verification model again wrongly predicts a negative correlation, with some subjects following a "predictive" strategy to produce larger facilitation and little inhibition, and others, an "expectancy" strategy to produce mostly inhibitory effects.

The original two-process model, of course, wrongly predicts a positive correlation within environment, with the attentional focusing producing part of the facilitation and all of the inhibition. However, the incongruity effect in the modified version of the model would produce inhibition that could be independent of attentional expectancies. The presence of inhibition in the low-congruity condition, and the absence of a positive correlation of facilitation and inhibition even in the high-predictability condition, suggests that such a strategy-free, "messagelevel" (Forster, 1979) incongruity effect was the primary source of contextual inhibition in Experiment 1.

One other dissociation between facilitation and inhibition was the tendency for facilitation to increase from the first to the second block of trials, and for inhibition to decrease. This trend was observed in each of the three environments, and was not due to differential changes in the neutral trial latency (see Table 2). The verification model is consistent with this pattern of change across blocks, as well as with the substantially larger increase in facilitation for the high-predictability condition than in the other validity conditions.

On balance, though, the effects of sentence validity were more consistent with predictions of the modified twoprocess model than with those of the verification model.

\section{EXPERIMENT 2}

The second experiment concerned the continued presence of inhibition in the low-congruity condition of Experiment 1. In terms of the environmental "metacontext," there are three possible reasons for the maintenance of inhibition under these conditions. First, the inclusion of even infrequent but highly predictable words may make it difficult to treat the context and test word as wholly unrelated, as was apparently possible in the case in which no tests were congruous or predictable (Fischler \& Bloom, 1979, Experiment 2). Second, it may be that the use of highly constraining sentence contexts in all conditions was the driving factor in the maintenance of contextual effects. Third, inhibition may occur whenever congruous and incongruous trials are mixed together, even if the predictability and constraint are low.

Two groups were created in Experiment 2. The sentence contexts for the high-constraint condition were those used in Experiment 1. In contrast to the balanced group of Experiment 1, all the likely completion test words were replaced by unlikely but congruous words, so that although the proportion of congruous trials and the level of constraint were the same as those of the balanced group, particular completions could not be predicted. A second set of contexts was developed for the low-constraint group, in which the predictability of the primary completions was much lower. Again, no primary completion was ever shown in context, and the proportion of congruous trials was equal to that for the high-constraint group. The nature of these two groups is outlined in Table 1 . 
Table 4

Mean Lexical Decision Latency (in Milliseconds) and Error Rates (ERs) for Two Constraint Environments of Experiment 2

\begin{tabular}{|c|c|c|c|c|c|c|c|}
\hline & \multicolumn{7}{|c|}{ Constraint Environment } \\
\hline & \multicolumn{3}{|c|}{ High Constraint } & \multicolumn{4}{|c|}{ Low Constraint } \\
\hline & \multirow{2}{*}{$\frac{\text { Unlikely }}{\text { Mean ER }}$} & \multicolumn{2}{|c|}{ Incongruous } & \multicolumn{2}{|c|}{ Uniikely } & \multicolumn{2}{|c|}{ Incongruous } \\
\hline & & Mean & ER & Mean & ER & Mean & ER \\
\hline \multicolumn{8}{|l|}{ Block 1} \\
\hline Context & 6124.0 & 639 & 9.5 & 609 & 4.5 & 686 & 7.5 \\
\hline Neutral & $566 \quad 4.3$ & 571 & 4.0 & 609 & 2.8 & 612 & 3.5 \\
\hline Context Effect & -46 & \multicolumn{2}{|c|}{-68} & \multicolumn{2}{|c|}{0} & \multicolumn{2}{|c|}{-74} \\
\hline \multicolumn{8}{|l|}{ Block 2} \\
\hline Context & $543 \quad 3.5$ & 592 & 8.5 & 590 & 2.5 & 648 & 4.0 \\
\hline Neutral & $540 \quad 1.5$ & 539 & 2.5 & 599 & 3.8 & 575 & 0.5 \\
\hline Context Effect & -3 & \multicolumn{2}{|c|}{-53} & \multicolumn{2}{|c|}{9} & \multicolumn{2}{|c|}{-73} \\
\hline Mean Effect & -24 & \multicolumn{2}{|c|}{-61} & \multicolumn{2}{|c|}{5} & \multicolumn{2}{|c|}{-74} \\
\hline
\end{tabular}

Note-Context effect is neutral minus context latency, so a negative effect indicates contextual inhibition.

\section{Method}

A second set of 100 sentence contexts, drawn from the Bloom and Fischler (1980) norms, had a probability of the primary completion of less than .55 (minimum .09, mean $p=.37$ ). These served as the sentence contexts for the low-constraint group. Sentence contexts from Experiment 1 were used for the high-constraint group. Each sentence context in the low-constraint set was associated with an unlikely test word, whose predictability in that context was less than 15 (mean $p=.02$ ), and an incongruous test word. As before, mean length, number of syllables, and frequency of occurrence of the two types of test words were roughly balanced. Two nonword stimuli were generated for each context by changing a single letter of the unlikely and incongruous words to produce a pronounceable pseudoword.

Context condition (sentence or neutral) and test type were varied within subject. The two different sets of contexts defined the highconstraint and low-constraint groups. In both groups, a 6:4 ratio of words to nonwords was maintained within blocks, identical to that of Experiment 1. Other aspects of the design, including the use of alternating context and neutral blocks and the assignment of particular contexts to the various test conditions, were the same as in Experiment 1. The apparatus and procedure for the two experiments were also identical. A total of 40 additional subjects participated in the experiment, 20 being assigned to each group according to order of appearance in the laboratory.

\section{Results}

Mean latency of correct responses was obtained for each subject in each context-test condition, and was used as a single score in subsequent analyses. As before, separate analyses were performed on the data for word and for nonword trials, with a .05 significance level used for the initial ANOVAs and a .01 level for post hoc tests.

Responses to words. Response latencies and error rates for word test trials are presented in Table 4. In the ANOVA, the main effect of block was significant $[F(1,38)$ $=11.89 \mathrm{j}$ but did not interact with other factors, and will not be discussed further. The important trends in Table 4 are the marginal effects of sentence contexts of the unlikely words and the consistent inhibition for incongruous words in both groups. Both the two-way interaction of context and test type $[F(1,38)=40.25]$ and the threeway interaction of context, test type, and environment $[F(1,38)=5.87]$ were significant. The latter interaction was produced by the greater discrepancy in context effects for unlikely-and incongruous-word latency for the low-constraint group. Simple effect tests showed that the inhibition for incongruous words in context was significant and equivalent for the two groups. For the unlikely words, neither the 5-msec facilitation for the lowconstraint nor the 24-msec inhibition for the highconstraint group was significant.

For error rates to word tests, the only significant effect was the interaction of context and test type, with a nonsignificant inhibition for unlikely words $(3.6 \%$ vs. $3.0 \%)$ and a significant contextual inhibition for incongruous words ( $7.4 \%$ vs. $2.6 \%$ ). The error rates show no sign of a trade-off associated with the inhibition for incongruous word trials.

Responses to nonwords. As with responses to words, nonword decision latency decreased with block $[F(1,38)$ $=6.88 \mathrm{l}$. Also, although the subjects in the high-constraint group were generally faster, there was no effect of context type ( $693 \mathrm{msec}$ for sentence contexts, $698 \mathrm{msec}$ for neutral contexts) and no interaction of context and environmnent. Error rate for nonwords was somewhat lower following sentences than following neutral contexts, however $(4.6 \%$ vs. $6.4 \%)[\mathrm{F}(1,38)=4.45, \mathrm{p}<.05]$.

\section{Subject Reports of Validity}

As in Experiment 1, the subjects were asked to estimate the proportions of predictable and congruous completions. Their mean estimates are shown in Table 5. A comparison of these estimates with those of Experiment 1 (Table 3) reveals a similar pattern: Estimates of congruity are reasonably accurate but are lower than the ac-

Table 5

Subjects' Estimates of the Proportion of Congruous and of Predictable Word Tests in Experiment 2

\begin{tabular}{lllc}
\hline & & \multicolumn{2}{c}{ Constraint Environment } \\
\cline { 4 - 4 } Probability of words that were congruous in context & Reported & .42 & .45 \\
& Actual & .67 & .67 \\
Predictability of words in context & & .22 & .30 \\
& Reported & .00 & .00 \\
\hline
\end{tabular}

*Probability of likely word given word $\times$ cloze probability of the likely word. 
tual proportion presented, and those of predictability are not as accurate, in this case being substantially higher than the actual proportions.

\section{Discussion}

The inhibition obtained for the high-constraint group (60 msec) is close to that for the balanced group ( $72 \mathrm{msec})$ in Experiment 1, so that the presence or absence of highly predictable test words appears to have had little effect on inhibition for incongruous words. Similarly, the 74-msec inhibition for the low-constraint group shows that the overall level of sentence constraint has little impact on the size of the incongruity effect. This outcome reinforces two themes of the first experiment: first, that congruity and predictability are distinct dimensions of sentencecontext effects; and second, that within broad bounds, changes in experiment-wide contextual environment do not produce substantial changes in these context effects.

The equivalence of inhibition for these three conditions suggests that its occurrence in the balanced and lowcongruity conditions in Experiment 1 was not due either to the occasional inclusion of predictable test words or to the overall high level of sentence constraint. There is also no suggestion in the data that when constraint and predictability are uniformly low, words that are congruous but unlikely receive any benefit from sentence contexts.

There is little support in the results of Experiment 2 for either the verification or the two-process models' predictions regarding shifts in expectancy resulting from experiment-wide changes in constraint. Since the cue validity in both environments was low because of the absence of likely completions, the two-process model would predict no inhibition for incongruous words in either environment. As in the predictions for Experiment 1, the verification model's predictions depend on whether high constraint or high predictability leads to the "prediction" strategy and subsequent facilitation dominance. The presence of inhibition for the high-constraint group clearly is at odds with the first possibility. And, as we have seen in the Experiment 1, inhibition was also obtained in the high-predictability environment. The results of Experiment 2 are most consistent with the modified two-process model, with inhibition produced by the incongruity of the sentence and test word as such, independently of particular expectancies.

\section{GENERAL DISCUSSION}

Since the major results of the experiments and their implications for two models of sentence-context effects have been discussed, we will provide a brief overview of these results here and then consider several recent studies that also have examined the role of contextual environment on sentence-context effects.

In both experiments, we found that subjects can estimate the average proportion of trials in which congruent words and, to a lesser extent, likely words had been shown following sentence contexts. This shows that the information about the contextual environment was available and could contribute to contextual effects. In an unpublished study, we have also found that subjects can accurately estimate the constraint that a given sentence context provides.

In contrast to the availability of this information, the actual effect of the contextual environment on contextual facilitation and inhibition was unimpressive. Only small changes in the facilitation for likely words were found when the proportion of these words was doubled, and congruity was equated, or when the proportion of congruent trials was halved (Experiment 1). The largest effect of environment was the increase in inhibition for incongruous words in the high-predictability versus the balanced environments of Experiment 1. A doubling of the proportion of congruent word trials had little effect on either facilitation or inhibition. The inhibition was also essentially unaffected by the elimination of any occurrence of likely-word tests (high-constraint environment, Experiment 2, vs. balanced environment, Experiment 1) or by a drastic change in the level of constraint provided by the sentences (low- vs. high-constraint environment, Experiment 2). Finally, there was little or no effect of sentence context on unlikely but congruous tests, regardless of the level of constraint or predictability.

In general, the pattern of results was more consistent with predictions of a two-process model than with those of the verification model. Across environments, the magnitude of facilitation and inhibition tended to be positively correlated, and within environments, facilitation and inhibition were simultaneously present. Moreover, inhibition increased, rather than decreased, when predictability of the test word increased. The greater effects of environment, such as they were, on inhibition for incongruent words than on facilitation for likely words are consistent with a relatively automatic priming of test words that are associatively related to words in the sentence context.

The embarrassments for the standard two-process model are the failure for facilitation to increase significantly along with inhibition when predictability was increased (high-predictability group, Experiment 1), the presence of inhibition without corresponding facilitation (both environments of Experiment 2; see also Fischler \& Bloom, 1980 ), the lack of effects of changes in proportion of congruity and in constraint on the amount of inhibition, and the absence of inhibition for unlikely but congruous words, especially in the presence of an equal number of likely test words with high contextual constraint (balanced environment, Experiment 1). It seems unlikely, given these results, that the "second of the two processes" is a conscious anticipation of one or several particular words to provide a benefit in response speed when validity is high.

As we have seen, most of these discrepancies can be explained if the inhibition results from a relatively auto- 
matic response to the implausibility of the test word in context (West \& Stanovich, 1982). Forster (1979) formalized this as the negative influence of the output of a message analyzer on the decision process. Since the language analyzers in this model are modular and autonomous, their activity should not be affected by such things as the experiment-wide proportion of congruous tests or by any conscious attempts to override them (see Fischler \& Bloom, 1979, Experiment 3). The one significant increase in inhibition observed here (high-predictability vs. balanced groups, Experiment 1) was accompanied by the only instance of the sentence contexts facilitating nonword latency, suggesting a shift in a decision criterion.

\section{Other Studies of Contextual Environment}

Several very recent studies have resembled the present series of experiments in observing the effects of different experiment-wide contextual environments on the facilitation and inhibition provided by sentence contexts. Two of these (Schwanenflugel \& Shoben, 1985; Sanocki \& Oden, 1984) have used a lexical decision task, and a third (Becker, 1982) used a naming task.

In the first three experiments of Schwanenflugel and Shoben (1985), all word tests were congruent with preceding sentence contexts. Among these, contexts of high (mean $\mathrm{p}=.85$ ) and low (mean $\mathrm{p}=.45$ ) constraint were followed by the most likely word, an unlikely word that was related in meaning to the likely word, or a nonword. When level of constraint was mixed (Experiment 1), highconstraint contexts showed substantial facilitation for likely words $(118 \mathrm{msec})$ and insignificant facilitation for unlikely words (23 msec); low-constraint sentences showed marginal facilitation for both "likely" and unlikely test words. Presenting only high-constraint contexts (Experiments 2 and 3) had little effect on likely-word facilitation (124 msec in Experiment 2) but produced a significant inhibition for unlikely words $(130 \mathrm{msec}) \mathrm{com}$ pared with the neutral (xx) control trials. Presenting only low-constraint sentences (Experiment 3) had little effect on the amount of facilitation seen in Experiment 1. Finally, introducing incongruous word completions in Experiment 4 for the mixed-constraint sentences gave significant inhibition for incongruous tests; this inhibition was greater for the high-constraint than for the lowconstraint contexts. Inclusion of incongruous tests increased only slightly the facilitation for likely and unlikely tests seen in the first study.

The results of the Schwanenflugel and Shoben (1985) study are in large part consistent with our own. Changes in the overall level of constraint had no effect on the sizable facilitation for very likely test words following highly constraining sentences, and the presence or absence of incongruous completions had little effect on either this facilitation or the smaller facilitation observed for unlikely test words.

The major difference between their results and ours was that in the present experiments, the unlikely test words showed no influence of sentence contexts in any of three different environments; in contrast, Schwanenflugel and Shoben (1985) were able to produce inhibition for unlikely words when constraint was high, facilitation when constraint was low, and no effect when constraint was mixed. The greater inhibition observed for high- than for lowconstraint contexts within the mixed environment in their Experiment 4 also contrasts with the absence of such a difference across environments in our Experiment 2 . It should be noted that these results are more consistent with the two-process model than with the verification model, and in fact are more supportive of the former than are our own data.

Typically, there are a number of areas of difference between the two studies that could produce the observed discrepancies. Their use of unlikely words that were related in meaning to the likely tests is one obvious example (see Kleiman, 1980), but why this would produce greater facilitation for these words with low-constraint sentences would be unclear. The overall level of constraint in the two studies is also very similar for both the high- and lowconstraint sentences. Compared with our data, those of Schwanenflugel and Shoben (1985) do show exaggerated effects of context in several instances; their facilitation for very likely words was more than twice ours, the inhibition for congruous tests was also twice that of our balanced environment, and they consistently found faster responses to nonwords in context than in isolation, whereas, in most cases, nonword latency showed no contextual effects in our study.

Two differences in procedure that could have produced larger attentional effects of context in their study were the subject-paced reading of contexts and the visual presentation of the test word at the end of the context string. Also, Schwanenflugel and Shoben's (1985) subjects were, on average, much slower for both word and nonword trials than were our subjects. The exaggerated effects of context with slower response latency is a common finding that is one of the main results in support of the "timelocked" aspect of the two-process model (see Stanovich \& West, 1983).

A similarly exaggerated effect of changes in environment on unlikely test words was reported by Sanocki and Oden (1984), who presented sentence contexts of very low constraint followed by a word or a nonword. In one environment, word tests were always congruous, and in a second environment, incongruous and congruous test words were equally likely. Sentence contexts facilitated response to unlikely words in the absence $(67 \mathrm{msec})$ but not in the presence $(-19 \mathrm{msec})$ of incongruous words. The greater effect of including incongruous-word trials on the context effect for unlikely words, compared with that in Schwanenflugel and Shoben (1985), may be attributable to the exclusion of any high-constraint contexts and likely test words in Sanocki and Oden's design. Their procedure also allowed self-paced reading of the contexts, 
with a full second between the completion of reading and the presentation of the test item; and again, the overall response times were substantially slower than those in the present study.

In both these recent lexical decision studies, we see somewhat larger effects of both sentence contexts and the environment in which they appear than in our study; and in both cases, the pattern of environment effects is more consistent with the predictions of the two-process model than with those of the verification model. A final report that must be considered is that of Becker (1982), who presented a set of strongly constraining $(p=.64)$ and weakly constraining $(p=.04)$ contexts among a larger set of either very high-constraint $(p=.94)$ or very lowconstraint $(\mathrm{p}=.00)$ filler contexts that determined the environment. Naming latency for words following the critical .64 and .04 contexts in the high-constraint environment showed mixed facilitation and inhibition for thirdand fifth-grade students and facilitation dominance for adults, similar to the results of West and Stanovich (1978). However, for the low-constraint environment, which, according to the verification model, should induce the inhibition-dominant expectancy strategy, the predicted pattern of inhibition without facilitation was obtained for both third-graders and adults. Besides being one of the few demonstrations of an inhibition of naming latency by sentence contexts in adults, the results of Becker's study constitute the strongest evidence in support of the verification model's predictions about effects of contextual environment on expectancy. The presence of context effects on unlikely words may be the result of Becker's procedure of inviting subjects to actively think of the words that could follow a given context. The specific direction of these effects is opposite to that observed by Schwanenflugel and Shoben (1985), and presents a challenge to the two-process explanation of expectancy effects in sentence priming.

On balance, however, studies of how the contextual "environment" modulates sentence-context effects suggest that, if response time is relatively rapid and the opportunities to generate expectancies are limited, effects due to the specific experiment-wide environment are correspondingly limited, even though the appropriate information may be available. This is consistent with Stanovich and West's (1983) recent argument that the higher level processing needed for comprehension of sentences reduces the resources available to utilize expectancies compared with what is possible for single-word primes. Since, during normal reading, such demands of comprehension will predominate, since incongruities will (it is hoped) be infrequent, and since word sequences high in associative strength are unlikely in typical prose passages, it would seem that none of the three main processes of contextual effects in the modified two-process model have much of a role to play in reading for meaning.

\section{REFERENCES}

BECKER, C. A. (1980). Semantic context effects in visual word recog- nition: An analysis of semantic strategies. Memory \& Cognition, $\mathbf{8}$, 493-512.

BECKER, C. A. (1982, November). Sentence context effects in children and adults. Paper presented at the meeting of The Psychonomic Society, Minneapolis, MN.

Bloom, P. A., \& Fischler, I.(1980). Completion norms for 329 sentence contexts. Memory \& Cognition, 8, 631-642.

De Groot, A. M. B., Thomassen, A. J. W. M., \& Hudson, P. T W. (1982). Associative facilitation as measured from a neutral prime Memory \& Cognition, 10, 358-370.

den Heyer, K., Briand, K., \& Dannenbring, G. L. (1983). Strategic factors in a lexical-decision task: Evidence for automatic and attention-driven processes. Memory \& Cognition, 11, 374-381.

Eisenberg, P., \& BeCKer, C. A. (1982). Semantic context effects in visual word recognition, sentence processing, and reading: Evidence for semantic strategies. Journal of Experimental Psychology: Human Perception and Performance, 8, 739-756.

FISCHLER, I. (1985). Word recognition, use of context, and reading skill among deaf college students. Reading Research Quarterly, 20, 203-218.

FisChlER, I., \& BLOOM, P. A. (1979). Automatic and attentional processes in the effects of sentence contexts on word recognition. Journal of Verbal Learning and Verbal Behavior, 18, 1-20.

FisChLER, I., \& BLOOM, P. A. (1980). Rapid processing of the meaning of sentences. Memory \& Cognition, 8, 216-225.

FORSTER, K. I. (1979). Levels of processing and the structure of the language processor. In W. E. Cooper \& E. Walker (Eds.), Sentence processing: Psycholinguistic studies presented to Merrill Garrett ( $\mathrm{pp}$. 27-85). Hillsdale, NJ: Erlbaum.

Foss, D. J. (1982). A discourse on semantic priming. Cognitive Psychology, 14, 590-607.

KLEIMAN, G. M. (1980). Sentence frame contexts and lexical decision: Sentence-acceptability and word-relatedness effects. Memory \& $\mathrm{Cog}$ nition, 8, 336-344

KUČERA, H., \& FranCIS, W.H. (1967). Computational analysis of present-day American English. Providence, RI: Brown University Press.

POSNER, M. I., \& SNyder, C. R. R. (1975). Attention and cognitive control. In R. L. Solso (Ed.), Information processing and cognition: The Loyola Symposium (pp. 55-85). Hillsdale, NJ: Erlbaum.

SANOCKI, T., \& ODEN, G. C. (1984). Contextual validity and the effects of low constraint sentence contexts on lexical decisions. Quart erly Journal of Experimental Psychology, 36A, 145-156.

Schuberth, R. E., \& Eimas, P. D. (1977). Effects of context on the classification of words and nonwords. Journal of Experimental Psychology: Human Perception and Performance, 3, 27-36.

SChuberth, R. E., SPOEhr, K. T., \& LANe, D. M. (1981). Effects of stimulus and contextual information on the lexical decision process. Memory \& Cognition, 9, 68-77.

SChWanenflugel, P. J., \& Shoben, E. J. (1985). The influence of sentence constraint on the scope of facilitation for upcoming words. Journal of Memory and Language, 24, 232-252.

Seidenberg, M. S., Waters, G. S., Sanders, M., \& Langer, P. (1984). Pre- and postlexical loci of contextual effects on word recognition. Memory \& Cognition, 12, 315-328.

Stanovich, K. E., \& WeSt, R. F. (1979). Mechanisms of sentence context effects in reading: Automatic activation and conscious attention. Memory \& Cognition, 7, 77-85.

StanOVICH, K. E. \& WEST, R. F. (1981). The effect of sentence context on ongoing word recognition. Journal of Experimental Psychology: Human Perception and Performance, 7, 658-672.

Stanovich, K. E., \& WEST, R. F. (1983). On priming by a sentence context. Journal of Experimental Psychology: General, 112, 1-36.

Stanovich, K. E., West, R. F., \& Feeman, D. J. (1981). A longitudinal study of sentence context effects in second-grade children: Tests of an interactive-compensatory model. Journal of Experimental Child Psychology, 32, 185-199.

TweEdy, J. R., Lapinski, R. H., \& SchVaneveldt, R. W. (1977) Semantic-context effects on word recognition: Influence of varying the proportion of items presented in an appropriate context. Memory \& Cognition, 5, 84-89. 
WESt, R. F., \& STANovich, K. E. (1978). Automatic contextual facilitation in readers of three ages. Child Development, 40, 717-727.

West, R. F., \& STanovich, K. E. (1982). Source of inhibition in experiments on the effect of sentence context on word recognition. Journal of Experimental Psychology: Learning, Memory, and Cognition, 8, 385-399.

\section{NOTE}

1. There is some controversy regarding the $\mathrm{XX}$ cue as a neutral baseline (see de Groot, Thomassen, \& Hudson, 1982, and Fischler \& Bloom, 1979). In general, it appears to slow lexical decision latency relative to a nonpredictive word (e.g., BLANK in de Groot et al.) in singleword priming studies, but be equivalent to a repeated word ("the, the ..") and a nonpredictive context ("They said it was the ...") in sentence-priming studies (Stanovich \& West, 1983, Experiment 2). Note that any such bias should be constant across environment in the present study and, further, would not affect any pattern of correlation between facilitation and inhibition scores

(Manuscript received July 2, 1984; revision accepted for publication January 21,1985 .) 